\title{
A Novel Framework of Health Monitoring Systems
}

\author{
Sonam Gupta, Ajay Kumar Garg Engineering College, Ghaziabad, India \\ Lipika Goel, Ajay Kumar Garg Engineering College, Ghaziabad, India \\ Abhay Kumar Agarwal, Kamla Nehru Institute of Technology, Ratan Pur, India
}

\begin{abstract}
IoT plays an important role in the healthcare domain for improving the quality of patient care. To analyze the patients' healthcare data, a real-time health-monitoring system is required. The proposed framework in this work is cable of such monitoring and sending alerts on critical circumstances. In this framework, the use of IoT devices makes it possible. This is very helpful in taking care of especially old wards and children in the absence or their caretakers. The function of alerting the caretakers and to inform hospital in critical condition makes this system one of its kind. Readings of patient pulse rates are taken from the pulse rate sensor and the body temperature is measured by MAX30205, a temperature sensor. The data is collected through sensors and sent over the cloud servers. Linear regression is used for further analysis and prediction of pulse and temperature trend lines. Corresponding health repots will be sent to the nearby hospitals and registered mobile numbers. The framework is validated with real-time patient data, and prediction is made regarding the trends.
\end{abstract}

\section{KEYWORDS}

Cloud System, Diagnosis, Health Monitoring, Internet of Things, Machine Learning

\section{INTRODUCTION}

Healthcare is an important domain and there is a need for it to be updated with the recent and upcoming technology. Internet of Things or IoT has been developed by an amalgamation of Radio Frequency Identification (RFID)] and Wireless Sensor Network (WSN) technologies and an advanced integration with cloud computing. Thus it enables customers over the internet to communicate with the data, collect, store and interchange it. With the help of the IOT system one can track patient's health conditions and collect it on cloud.

This IoT based project intends to serve the patients as well as to aid the doctors with their diagnosis, thereby saving time. Since the system will be powered by commands and will capture the patient's data by using medical sensors, it makes the patient's data accessible to the doctor for review thereby reducing the doctor's labour time by about 15 hours. Moreover it helps reducing emergency room wait time because patient's medical history will be available to the doctor in prior for review.

It makes use of technologies like Machine Learning which uses diagnostic algorithms to derive some major conclusions about a patient's health from the data provided like pulse rate and body temperature. Cloud Computing is a technology used to store large amount of data over the internet. In this project, it is used to store the patient's diagnostic data into a large database. The readings from pulse rate sensor or temperature sensor are all put up on a cloud and made available to doctor, 
patient or can be provided to machine learning algorithms to perform diagnosis. With IOT, doctors and other medical associates may be able to know about the status of patient, and can save it on their database for further accessing and analysis.

\section{STATE OF ART}

In this era of Technology Internet of Things or popularly also known as IoT along with Cloud Computing play a very important role in monitoring the health condition of a person using various kinds of data collected using body sensors fitted in different types of wearable devices. It mainly uses Raspberry Pi board for this purpose. The collected data is processed using various machine learning algorithms and hence processed data is used to detect diseases and preventions that need to be taken. This data is then sent to the patients as well as concerned people and doctors to take recommended actions for precautionary purposes. As this data is kept on the cloud it can also be accessed anywhere and anytime around the world. It is very suitable in areas where medical facilities are not easily available, areas like villages or rough terrains. Body wireless sensor Network (BWSN) is used to transmit the patients' health parameters collected through Raspberry Pi microcontroller to the physicians and caretaker wirelessly. As we know that these devices are long ranged due to high bandwidths it is really easy and efficient to detect the patient's location in case of any emergency and timely intervention of responsible people, the server also rings alarm to nearby ambulance while itself performing various basic health care check-ups by continuously monitoring various body parameters and reporting the respective data. Use of smart devices increases the data accuracy and data protection on various parameters. The historical data uploaded on the cloud can also be used to perform various tests and at times infer data from family history's data or the health history of the patient himself.

Universal Health care concern and its solution has become crucial nowadays as it is necessary to pay attention to awareness on Healthcare. Mobile devices are available to us nowadays very easily. These devices help to collect data from the wearables and analyse it under a wireless sensor network. This reduces complications regarding location problems etc. We can also make use of Barcode Reader to verify and aid our patients.

With the increased advancement of the sensors and wearables, it makes the health control and management system more powerful in remote areas. Now this needs to be secure. Our system designs are planted upon the WSN which is Wireless Sensor Networks. These systems have reduced the cost as the doctors can analyse the patient's health without actually visiting them for small health issues by collecting his data and sending the instructions to get cured.

An android app has been created for healthcare authority called ECG App, which provides the client with the Electro Cardiogram Waves and his/her data. This data gets uploaded to the cloud of the client which can be further analysed by the patients and doctors. This system is helpful for all whether they are at the hospital or at their home.

Gomez et al. (2016) showed that the Universal Health care concern and its solution has become crucial nowadays as it is necessary to pay attention to awareness on Healthcare. Mobile devices are available to us nowadays very easily. These devices help to collect data from the wearables and analyse it under a wireless sensor network. This reduces complications regarding location problems etc. We can also make use of Barcode Reader to verify and aid our patients.

With the increased advancement of the sensors and wearables, it makes the health control and management system more powerful in remote areas. Now this needs to be secure. Our system designs are planted upon the WSN which is Wireless Sensor Networks. These systems have reduced the cost as the doctors can analyse the patient's health without actually visiting them for small health issues by collecting his data and sending the instructions to get cured.

An android app has been created for healthcare authority called ECG App, which provides the client with the Electro Cardiogram Waves and his/her data. This data gets uploaded to the cloud of 
the client which can be further analysed by the patients and doctors. This system is helpful for all whether they are at the hospital or at their home.

Jorge Gomez et al. (2016) on the basis of evidence and symptoms of the patients, made analysis. By continuously monitoring the issues and the characteristics of the patient, and also of the improvement of the system, Franca told that these fruits of the innovations of the new generation devices and systems. He also focused on the use of wireless technologies which would help in increasing productivity. Tartarisco et al. (2012) inferred that lack of proper treatment on time, can lead to serious issues and sometimes death of the patients, that's why a health monitoring system with proper solutions of the problem is a need. Sivakanth et al. (2016) proposed the idea of collecting data based on various geographic locations. The data will be sent to the doctors nearby the patient's location and treatment could be done. Pustiek et al. (2015) showed that, IOT techniques which may be helpful in improving and to support healthcare. Data in heterogeneous formats will be helpful in creating real time applications. He also suggested that the patient should be made alert based on their fitness, past treatment and also heredity related issues.

Franca Delmastro et al. (2012) stated that the data transmitted in the monitoring systems by the wearables and sensors, needs to be in heterogeneous formats so that the user can access the data in the desired form and which in turn can be useful in building real time applications and time to time update is also necessary in the user's application. Nichal et al. (2015) discussed the important information of the software for healthcare and gave an suitable prototype for healthcare and IOT. He has taken the parameters like ECG, oxygen amount in blood, respiration, temperature etc. As there remains a lot many achievements and pros to this invention there are a very few factors that make this device a bit challenging. First being it's compulsory to have a running internet connection to efficiently run this device. Second challenge is the accuracy and validity of the data collected which can at very bleak chances can go wrong due to machine error or young training of the model at the start of this setup. Third could be the need for continuous wearing of this device for proper assessing of data and good amount of data to be collected for more accurate processing of the information.

But all in all these challenges have very less importance in front of the effectiveness, ease and feasibility that is offered by the device making the health care system more efficient and reachable by all people in the society.

New challenges and requirements need to be handled in the view to make the IoT future. Many challenges like the internet connectivity, the proper allowed distance between the sensors and the body parts etc. need to be focused upon. The system must be secure enough to handle absurd situations.

\section{PREREQUISITE KNOWLEDGE}

\subsection{Linear Regression}

Linear Regression, a well known supervised machine learning algorithm which has its slope constant and the predicted output continuous. This algorithm does not classify the values into categories but predict values within a specified continuous range. It works to find the relationship between dependent variables and set of independent variables. This means that when a set of one or more independent variables(x) changes, the values for dependent variables(y) may also be affected accordingly. There is a linear relationship between the independent(x) and dependent $(\mathrm{y})$ variable. The red line in the graph is the best fit straight line. There are two main types:

- Simple Regression: Simple linear regression uses traditional slope-intercept form, where m and $\mathrm{c}$ are the variables, the algorithm will try to learn, $\mathrm{x}$ is the input data to be fed and $\mathrm{y}$ is the prediction: 
$y=m x+c$

where:

$\mathrm{y}=\mathrm{a}$ function of $\mathrm{x}$

$\mathrm{m}=$ gradient, angle of the line to $\mathrm{x}$-axis

$\mathrm{c}=$ is intercept on $\mathrm{y}$-axis

- Multivariable Regression: A more complex, multi-variable linear equation might look like this, where $w$ represents the coefficients, or weights, our model will try to learn:

$f(x, y, z)=w 1 x+w 2 y+w 3 z$

The variables $\mathrm{x}, \mathrm{y}, \mathrm{zx}, \mathrm{y}, \mathrm{z}$ represent the attributes, or distinct pieces of information, we have about each observation.

The formulas related to linear regression are as follows:

Slope $\mathrm{b}=\sum\left(\mathrm{Y}_{\mathrm{i}}-\bar{Y}\right)\left(\mathrm{X}_{\mathrm{i}}-\bar{X}\right) / \sum\left(\mathrm{X}_{\mathrm{i}}-\bar{X}\right)^{2}$

Variance $\sigma^{2} / \sum\left(\mathrm{X}_{\mathrm{i}}-\bar{X}\right)^{2}$

Intercept $\mathrm{a}=\bar{Y}-\mathrm{b} \bar{X}$

Variance of a $\left[\frac{1}{n}+\frac{X^{2}}{\sum(X i-\bar{X})^{2}}\right] \sigma^{2}$

Estimated mean at $\mathrm{X}_{0} \mathrm{a}+\mathrm{bX} \mathrm{X}_{0}$

Variance $\left[\frac{1}{n}+\frac{(X 0-\bar{X})^{2}}{\sum(X i-\bar{X})^{2}}\right] \sigma^{2}$

Estimated individual at $\mathrm{X}_{0} \mathrm{a}+\mathrm{b} \mathrm{X}_{0}$

Variance $\left[1+\frac{1}{n}+\frac{(X 0-\bar{X})^{2}}{\sum(X i-\bar{X})^{2}}\right] \sigma^{2}$

Total SS $=\sum\left(\mathrm{Y}_{\mathrm{i}}-\bar{Y}\right)^{2}$

Regression $\mathrm{SS}=\left[\sum\left(\mathrm{Y}_{\mathrm{i}}-\bar{Y}\right)\left(\mathrm{X}_{\mathrm{i}}-\bar{X}\right)\right]^{2} / \sum\left(\mathrm{X}_{\mathrm{i}}-\bar{X}\right)^{2}$

Error SS $=$ Total SS - Regression SS

$\mathrm{R}^{2}=$ Regression SS / Total SS = "proportion explained"

$\mathrm{MSE}=$ error mean square $=$ estimate of $\sigma^{2}=$ Error SS $/ \mathrm{df}$

$\mathrm{df}=$ degree of freedom $=\mathrm{n}-2$ for sample linear

\section{PROPOSED FRAMEWORK}

The project focuses to resolve one of the pressing problem we face in the health care system that is of diagnosis and critical time management. It uses a healthcare system developed around the latest technology of IoT. The proposed framework is mentioned in Figure 1. 
Readings of patient's pulse rate are taken from the pulse rate sensor and the body temperature is measured by MAX30205, a temperature sensor. Temperature sensor and pulse rate sensor are external devices used to measure the patient's details. These send out the data to NodeMCU. NodeMCU is an open source LUA based firmware developed for ESP8266 wifi chip. It is an open source platform which means that its hardware is open for editing, modifying and building. NodeMCU Development board is featured with wifi capability, analog pin, digital pins and serial communication protocols. This healthcare device is connected to an IoT cloud server. The data stored in the cloud server can be reused in the future for reference and analysis as well. A summary report of the patient will be sent to the registered email id. Alerts in the critical conditions will be sent to the registered phone numbers.

The results received by NodeMCU are then analysed using some strict and unique algorithms. Linear Regression is used to determine the trend line. The trend of the patients pulse rate and temperature is used to generate reports for the user and doctor. They reduce the labour work and time of doctors as well as hospital management. The IoT cloud server has a database in connection with a web portal. With unique data analytics algorithm which is capable of diagnosing ailments from the statistical data provided to them through the external healthcare device system. The web portal gathers all the data, stores stored at same place and is responsible for generating and sending out a copy of the report through email to the doctor as well as the guardian/patient.

\section{RESULT}

The pulse and the temperature data values as recorded by the IOT system is tabulated in Table 1 . Figure 2 and 3 plots its corresponding pulse and temperature graphs. The predicted trends of pulse and temperature values are tabulated in Table 2 and 4 respectively. Table 3 and Table 5 states the statistical values of Linear Regression on Pulse and Temperature data values. Figure 4 and 5 are the prediction trends of pulse and temperature respectively. Figure 6 is the sample diagnosis report of the patient which will be available on his/her login. This report can be mailed to the doctor as well so that during emergency the basic tests of the patient need not to be done again and again.

The following observations are made:

Figure 1. The framework

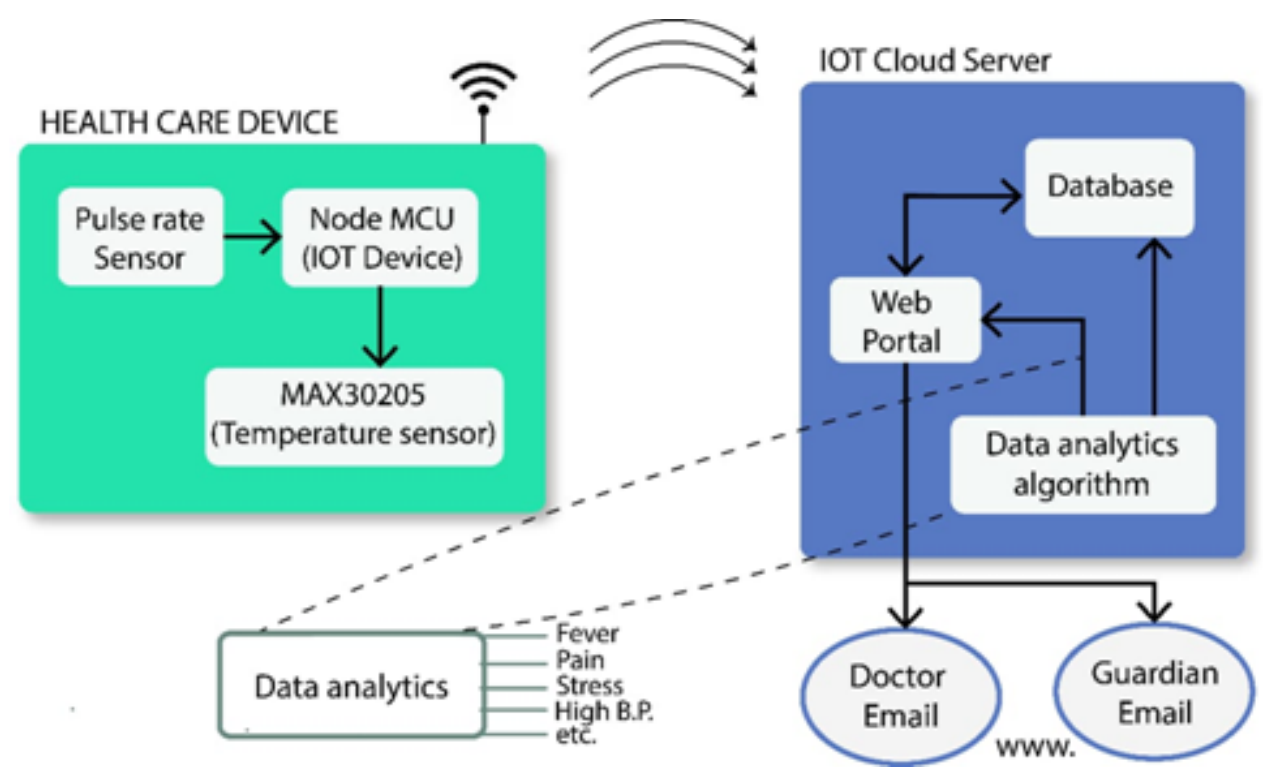


- As time interval and Pulse/Temperatur data points are independent, we should not be predicting the pulse/temperature values based on time intervals;

- As data points are independent, we should not be driven any reference point of Sign. F/P-values based on time interval, as the statistical outcomes are not up to the mark to describe it as a good model;

- We are using linear regression to see the trend of data to get the tentative idea of the trend of values along with the time interval;

- It is looking similar to moving average of data values, where the trend can be plotted based on initial benchmarks, like based on initial 5 timelines, subsequent data trendline can plot similar to the linear regression trend line.

\section{THREATS TO VALIDITY}

In this paper, the treats to validity is discussed under the following threats:

- Threats to internal and external validity

- Threats to reliability validity

- Threats to conclusion validity

The experimental errors are considered under the treats to internal validity. There might be some unnoticed errors although the datasets and the experiment have been examined carefully. The machine learning includes many algorithms. We have used Linear Regression in our experiment. The usage of limited classifier for prediction and modeling can affect the internal validity. The external validity generates due to generalization of the results. On consideration of more datasets from different sources and patients may reduce the threat of external validity.

The stated work has the probability of replication thereby causing the threat to reliability validity.

The threat to conclusion validity is that we have analysed our outcomes of the experiment on the basis of trend line plotted using Linear Regression The proposed model can be also compared with the other baseline framework in the state of art of CPDP.

\section{CONCLUSION}

Healthcare is an important domain and there is a need for it to be updated with the recent and upcoming technology. The IOT based health monitoring system is an emerging service in the healthcare domain. It has the potential to bring revolution in the healthcare and medical industry. It improves the availability of the patients healthcare data. Continuous monitoring and real time analysis for tracking the patients health is made easy through IOT and Machine Learning.

This is very helpful in taking care of especially old wards and children in the absence or their caretakers. Health Data management of patient at cloud server can make it paperless, which is the need of the hour. Its added advantage is that it will reduce hospital's management cost with the help of an IOT based mobile app and we can expect this to reduce the patient's treatment charges making healthcare sector affordable to many.

Moreover it helps reducing emergency room wait time because patient's medical history will be available to the doctor in prior for review.

Future work will involve implementation of GPS sensor on the framework so that the current location of the patient can be tracked. Immediate actions can be taken in catastrophic conditions. Moreover, usage of the GPS sensor will enable to locate the nearby hospitals. This in turn will assist in critical and emergency situations. 
Table 1. Pulse and temperature data values

\begin{tabular}{|c|c|c|c|c|c|}
\hline Interval & Pulse & Temp & Interval & Pulse & Temp \\
\hline 1 & 73 & 36 & 30 & 75 & 33 \\
\hline 2 & 78 & 37 & 31 & 87 & 32 \\
\hline 3 & 74 & 32 & 32 & 90 & 30 \\
\hline 4 & 63 & 36 & 33 & 86 & 32 \\
\hline 5 & 69 & 30 & 34 & 85 & 40 \\
\hline 6 & 77 & 37 & 35 & 70 & 35 \\
\hline 7 & 66 & 31 & 36 & 72 & 30 \\
\hline 8 & 72 & 36 & 37 & 78 & 35 \\
\hline 9 & 85 & 37 & 38 & 69 & 34 \\
\hline 10 & 65 & 35 & 39 & 66 & 37 \\
\hline 11 & 84 & 34 & 40 & 82 & 32 \\
\hline 12 & 66 & 37 & 41 & 66 & 38 \\
\hline 13 & 72 & 37 & 42 & 60 & 34 \\
\hline 14 & 75 & 37 & 43 & 86 & 40 \\
\hline 15 & 67 & 40 & 44 & 61 & 38 \\
\hline 16 & 89 & 38 & 45 & 74 & 36 \\
\hline 17 & 73 & 37 & 46 & 85 & 40 \\
\hline 18 & 80 & 32 & 47 & 60 & 37 \\
\hline 19 & 88 & 30 & 48 & 81 & 38 \\
\hline 20 & 90 & 36 & 49 & 90 & 36 \\
\hline 21 & 90 & 37 & 50 & 83 & 31 \\
\hline 22 & 61 & 32 & 51 & 80 & 34 \\
\hline 23 & 87 & 36 & 52 & 85 & 32 \\
\hline 24 & 61 & 40 & 53 & 62 & 34 \\
\hline 25 & 62 & 39 & 54 & 77 & 39 \\
\hline 26 & 77 & 40 & 55 & 82 & 39 \\
\hline 27 & 64 & 31 & & & \\
\hline 28 & 64 & 30 & & & \\
\hline 29 & 88 & 31 & & & \\
\hline
\end{tabular}

\section{ACKNOWLEDGMENT}

This research is supported by APJ Abdul Kalam Technical University, Lucknow under Collaborative

Research Innovative Project by TEQIP-III. 
International Journal of Big Data and Analytics in Healthcare

Volume 6 • Issue 1 • January-June 2021

Figure 2. Graphical plot of the pulse data values

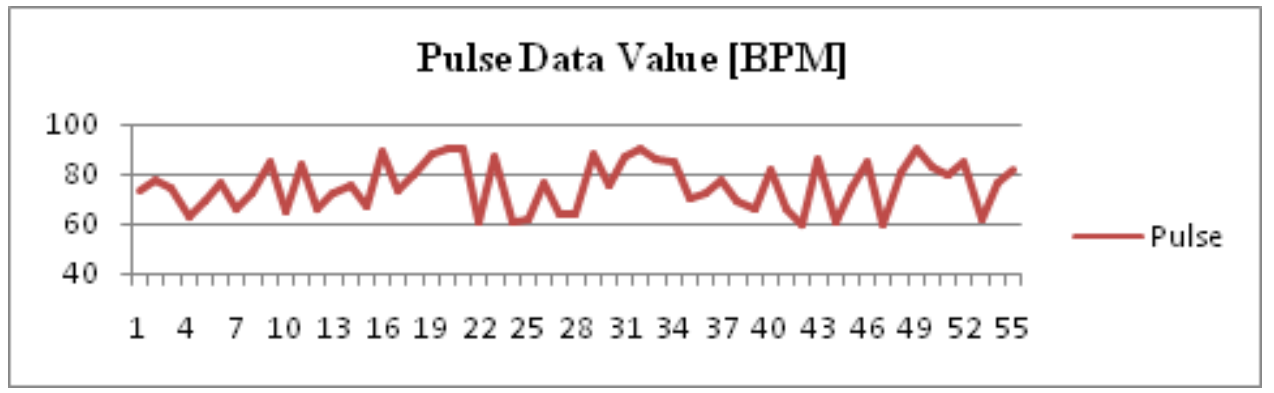

Figure 3. Graphical plot of the body temperature data values

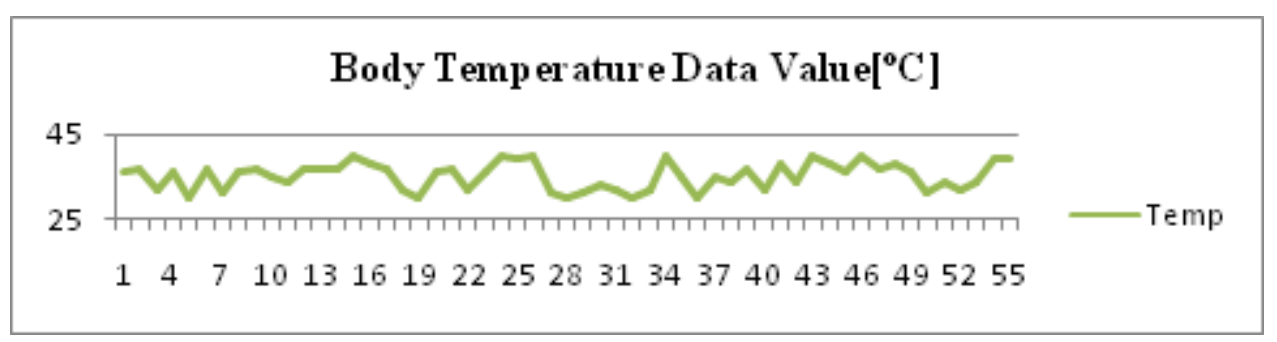


Table 2. Predicted trends of the pulse data values

\begin{tabular}{|c|c|c|c|c|c|}
\hline Interval & Pulse & Predicated Trends & Interval & Pulse & $\begin{array}{c}\text { Predicated } \\
\text { Trends }\end{array}$ \\
\hline 1 & 73 & 73.8525974 & 31 & 87 & 75.67294372 \\
\hline 2 & 78 & 73.91327561 & 32 & 90 & 75.73362193 \\
\hline 3 & 74 & 73.97395382 & 33 & 86 & 75.79430014 \\
\hline 4 & 63 & 74.03463203 & 34 & 85 & 75.85497835 \\
\hline 5 & 69 & 74.09531025 & 35 & 70 & 75.91565657 \\
\hline 6 & 77 & 74.15598846 & 36 & 72 & 75.97633478 \\
\hline 7 & 66 & 74.21666667 & 37 & 78 & 76.03701299 \\
\hline 8 & 72 & 74.27734488 & 38 & 69 & 76.0976912 \\
\hline 9 & 85 & 74.33802309 & 39 & 66 & 76.15836941 \\
\hline 10 & 65 & 74.3987013 & 40 & 82 & 76.21904762 \\
\hline 11 & 84 & 74.45937951 & 41 & 66 & 76.27972583 \\
\hline 12 & 66 & 74.52005772 & 42 & 60 & 76.34040404 \\
\hline 13 & 72 & 74.58073593 & 43 & 86 & 76.40108225 \\
\hline 14 & 75 & 74.64141414 & 44 & 61 & 76.46176046 \\
\hline 15 & 67 & 74.70209235 & 45 & 74 & 76.52243867 \\
\hline 16 & 89 & 74.76277056 & 46 & 85 & 76.58311688 \\
\hline 17 & 73 & 74.82344877 & 47 & 60 & 76.64379509 \\
\hline 18 & 80 & 74.88412698 & 48 & 81 & 76.7044733 \\
\hline 19 & 88 & 74.94480519 & 49 & 90 & 76.76515152 \\
\hline 20 & 90 & 75.00548341 & 50 & 83 & 76.82582973 \\
\hline 21 & 90 & 75.06616162 & 51 & 80 & 76.88650794 \\
\hline 22 & 61 & 75.12683983 & 52 & 85 & 76.94718615 \\
\hline 23 & 87 & 75.18751804 & 53 & 62 & 77.00786436 \\
\hline 24 & 61 & 75.24819625 & 54 & 77 & 77.06854257 \\
\hline 25 & 62 & 75.30887446 & 55 & 82 & 77.12922078 \\
\hline 26 & 77 & 75.36955267 & 56 & 84 & 77.18989899 \\
\hline 27 & 64 & 75.43023088 & 57 & 82 & 77.2505772 \\
\hline 28 & 64 & 75.49090909 & 58 & 86 & 77.31125541 \\
\hline 29 & 88 & 75.5515873 & 59 & 85 & 77.37193362 \\
\hline 30 & 75 & 75.61226551 & 60 & 85 & 77.43261183 \\
\hline
\end{tabular}


Table 3. Statistical values of linear regression on pulse data

\begin{tabular}{|l|l|l|l|l|l|l|l|l|}
\hline Regression Statistics & & & & & & & \\
\hline Multiple R & 0.100091 & & & & & & & \\
\hline R Square & 0.010018 & & & & & & & \\
\hline $\begin{array}{l}\text { Adjusted R } \\
\text { Square }\end{array}$ & -0.00866 & & & & & & & \\
\hline $\begin{array}{l}\text { Standard } \\
\text { Error }\end{array}$ & 9.754259 & & & & & & & \\
\hline Observations & 55 & & & & & & & \\
\hline ANOVA & & & & & & & & \\
\hline & df & SS & MS & F & Significance & & & \\
\hline Regression & 1 & 51.03038 & 51.03038 & 0.53634 & 0.467179944 & & & \\
\hline Residual & 53 & 5042.715 & 95.14557 & & & & & \\
\hline Total & 54 & 5093.745 & & & & & & \\
\hline & Coefficients & $\begin{array}{l}\text { Standard } \\
\text { Error }\end{array}$ & $\mathbf{t}$ Stat & P-value & Lower 95\% & $\begin{array}{l}\text { Upper } \\
\mathbf{9 5 \%}\end{array}$ & $\begin{array}{l}\text { Lower } \\
\mathbf{9 5 . 0 \%}\end{array}$ & $\mathbf{\text { Upper }}$ \\
\hline Intercept & 73.79192 & 2.666813 & 27.67045 & $3.53 E-$ & 68.44297 & 79.14087 & 68.44297 & 79.14086843 \\
\hline X Variable 1 & 0.060678 & 0.082854 & 0.732352 & 0.46718 & -0.10551 & 0.226862 & -0.10551 & 0.226861978 \\
\hline
\end{tabular}

Figure 4. Pulse data values prediction trends

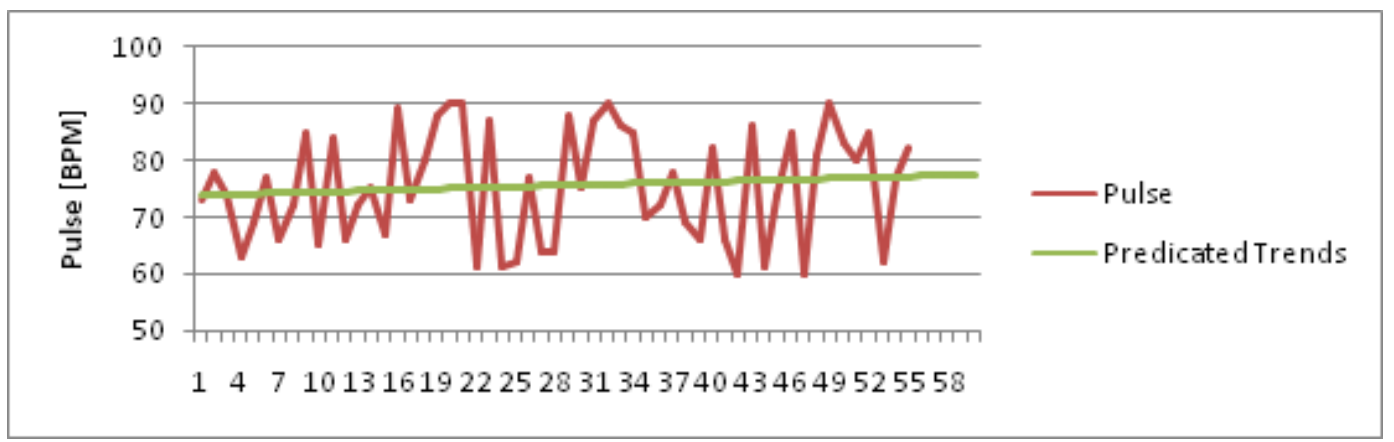


Table 4. Predicted trends of the temperature data values

\begin{tabular}{|c|c|c|c|c|c|}
\hline Interval & Temp & Predicated Temp & Interval & Temp & Predicated Temp \\
\hline 1 & 36 & 34.84220779 & 31 & 32 & 35.25996 \\
\hline 2 & 37 & 34.85613276 & 32 & 30 & 35.27388 \\
\hline 3 & 32 & 34.87005772 & 33 & 32 & 35.28781 \\
\hline 4 & 36 & 34.88398268 & 34 & 40 & 35.30173 \\
\hline 5 & 30 & 34.89790765 & 35 & 35 & 35.31566 \\
\hline 6 & 37 & 34.91183261 & 36 & 30 & 35.32958 \\
\hline 7 & 31 & 34.92575758 & 37 & 35 & 35.34351 \\
\hline 8 & 36 & 34.93968254 & 38 & 34 & 35.35743 \\
\hline 9 & 37 & 34.9536075 & 39 & 37 & 35.37136 \\
\hline 10 & 35 & 34.96753247 & 40 & 32 & 35.38528 \\
\hline 11 & 34 & 34.98145743 & 41 & 38 & 35.39921 \\
\hline 12 & 37 & 34.9953824 & 42 & 34 & 35.41313 \\
\hline 13 & 37 & 35.00930736 & 43 & 40 & 35.42706 \\
\hline 14 & 37 & 35.02323232 & 44 & 38 & 35.44098 \\
\hline 15 & 40 & 35.03715729 & 45 & 36 & 35.45491 \\
\hline 16 & 38 & 35.05108225 & 46 & 40 & 35.46883 \\
\hline 17 & 37 & 35.06500722 & 47 & 37 & 35.48276 \\
\hline 18 & 32 & 35.07893218 & 48 & 38 & 35.49668 \\
\hline 19 & 30 & 35.09285714 & 49 & 36 & 35.51061 \\
\hline 20 & 36 & 35.10678211 & 50 & 31 & 35.52453 \\
\hline 21 & 37 & 35.12070707 & 51 & 34 & 35.53846 \\
\hline 22 & 32 & 35.13463203 & 52 & 32 & 35.55238 \\
\hline 23 & 36 & 35.148557 & 53 & 34 & 35.56631 \\
\hline 24 & 40 & 35.16248196 & 54 & 39 & 35.58023 \\
\hline 25 & 39 & 35.17640693 & 55 & 39 & 35.59416 \\
\hline 26 & 40 & 35.19033189 & 56 & & 35.60808 \\
\hline 27 & 31 & 35.20425685 & 57 & & 35.62201 \\
\hline 28 & 30 & 35.21818182 & 58 & & 35.63593 \\
\hline 29 & 31 & 35.23210678 & 59 & & 35.64986 \\
\hline 30 & 33 & 35.24603175 & 60 & & 35.66378 \\
\hline
\end{tabular}


Figure 5. Temperature data values prediction trends

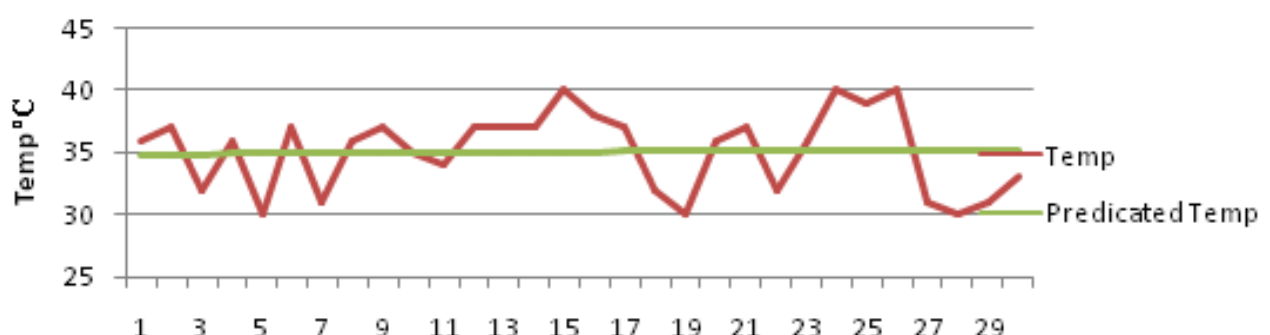

$\begin{array}{lllllllllllllll}1 & 3 & 5 & 7 & 9 & 11 & 13 & 15 & 17 & 19 & 21 & 23 & 25 & 27 & 29\end{array}$

Table 5. Statistical values of linear regression on temperature data

\begin{tabular}{|c|c|c|c|c|c|c|c|c|}
\hline \multicolumn{2}{|c|}{ Regression Statistics } & & & & & & & \\
\hline Multiple R & 0.070587 & & & & & & & \\
\hline R Square & 0.004983 & & & & & & & \\
\hline $\begin{array}{l}\text { Adjusted R } \\
\text { Square }\end{array}$ & -0.01379 & & & & & & & \\
\hline $\begin{array}{l}\text { Standard } \\
\text { Error }\end{array}$ & 3.182186 & & & & & & & \\
\hline Observations & 55 & & & & & & & \\
\hline \multirow[t]{2}{*}{ ANOVA } & & & & & & & & \\
\hline & df & SS & MS & $\mathbf{F}$ & $\begin{array}{l}\text { Significance } \\
\text { F }\end{array}$ & & & \\
\hline Regression & 1 & 2.687518 & 2.687518 & 0.2654 & 0.608577 & & & \\
\hline Residual & 53 & 536.6943 & 10.12631 & & & & & \\
\hline \multirow[t]{2}{*}{ Total } & 54 & 539.3818 & & & & & & \\
\hline & Coefficients & $\begin{array}{l}\text { Standard } \\
\text { Error }\end{array}$ & t Stat & P-value & Lower 95\% & $\begin{array}{l}\text { Upper } \\
95 \%\end{array}$ & $\begin{array}{l}\text { Lower } \\
95.0 \%\end{array}$ & $\begin{array}{l}\text { Upper } \\
95.0 \%\end{array}$ \\
\hline Intercept & 34.82828 & 0.870009 & 40.03209 & $2.73 \mathrm{E}-41$ & 33.08327 & 36.5733 & 33.08327 & 36.5733 \\
\hline $\mathrm{X}$ Variable 1 & 0.013925 & 0.02703 & 0.515169 & 0.608577 & -0.04029 & 0.06814 & -0.04029 & 0.06814 \\
\hline
\end{tabular}


Figure 6. Sample diagnosis report

\section{Diagnosis Report of Mr. Sandeep Gutpa}

Date \& Time 07-Feb-2020 22:21

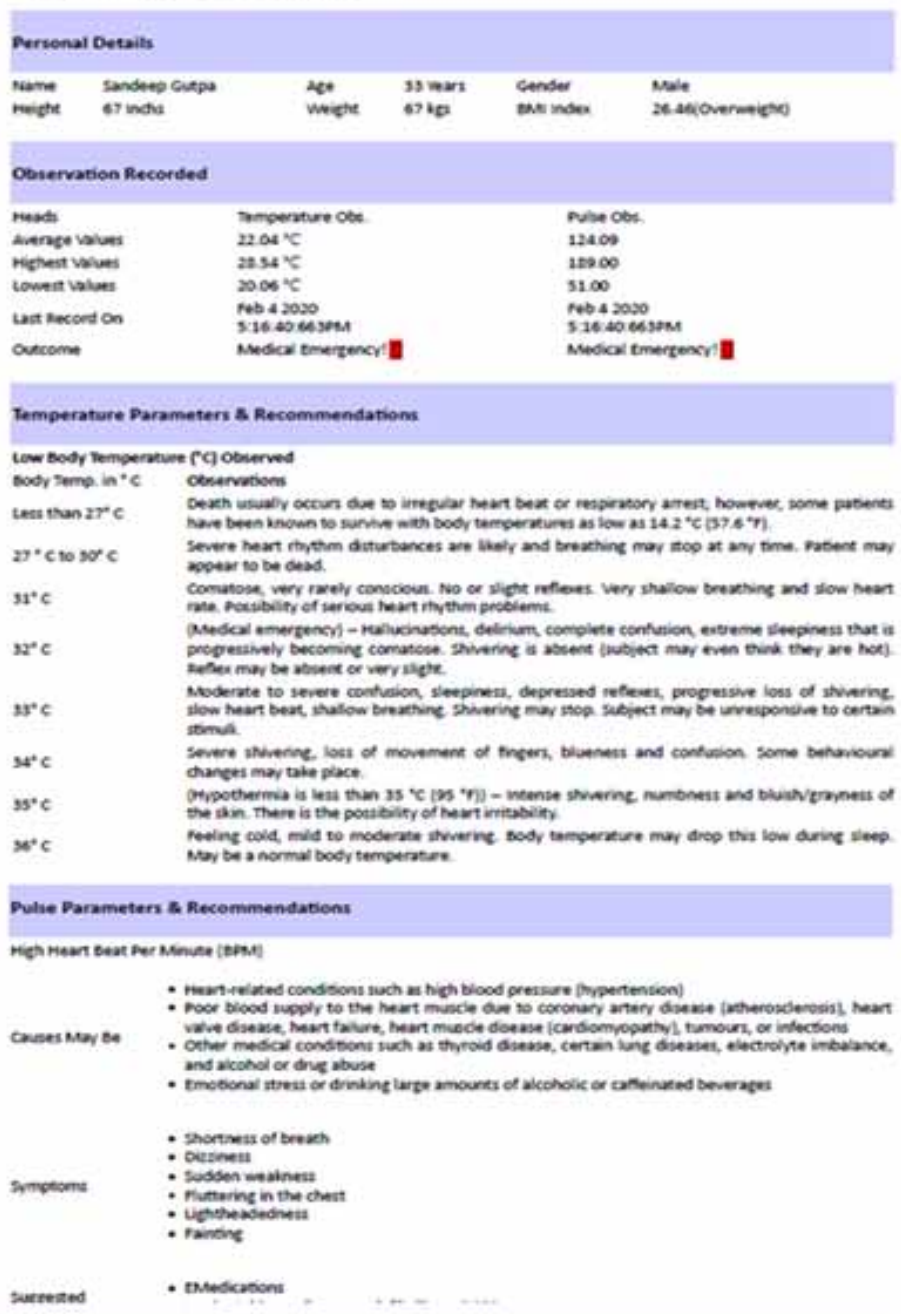

Pule Chart of tast 40-Obiervation

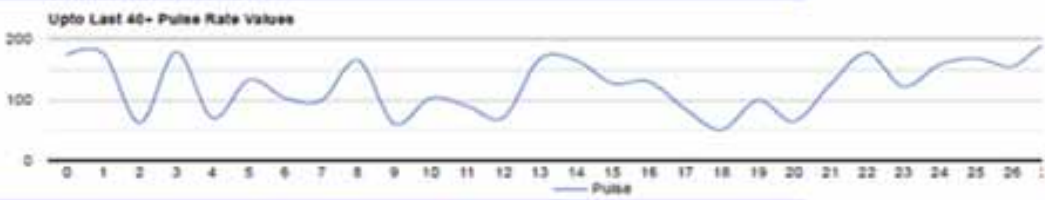

Temperature Chart of latt 40-Obiersatios

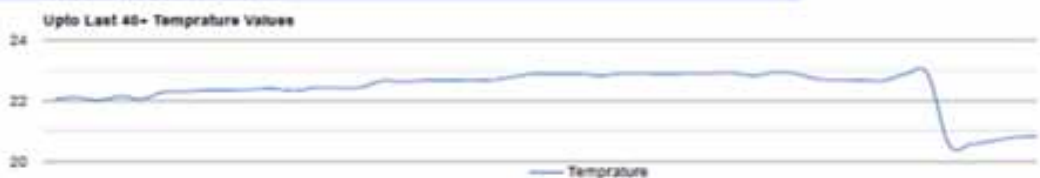




\section{REFERENCES}

Delmastro, F. (2012). Pervasive communications in healthcare. Computer Communications, 35(11), 1284-1295. doi:10.1016/j.comcom.2012.04.018

Gómez, J. E., Oviedo, B., \& Zhuma, E. (2016, December). Patient Monitoring System Based on Internet of Things. In ANT/SEIT (pp. 90-97). doi:10.1016/j.procs.2016.04.103

Nichal, S. N., \& Singh, J. K. (2015). Raspberry pi Based Smart Supervisor using Internet of Things (IoT). International Journal of Advanced Research in Electronics and Communication Engineering, 4(7), 1922-1926.

Pustiek, M., Beristain, A., \& Kos, A. (2015, October). Challenges in wearable devices based pervasive wellbeing monitoring. In 2015 international conference on Identification, Information, and Knowledge in the Internet of Things (IIKI) (pp. 236-243). IEEE. doi:10.1109/IIKI.2015.58

Sivakanth, T., \& Kolangiammal, S. (2016). Design of Iot based smart health monitoring and alert system. IJCTA, 9(15), 7655-7661.

Tartarisco, G., Baldus, G., Corda, D., Raso, R., Arnao, A., Ferro, M., Gaggioli, A., \& Pioggia, G. (2012). Personal Health System architecture for stress monitoring and support to clinical decisions. Computer Communications, 35(11), 1296-1305. doi:10.1016/j.comcom.2011.11.015

Sonam Gupta (PhD) is B.E., Mtech, and Ph.D. She has over 10 years of experience in teaching. Currently, she is working as an Associate Professor (CSE) in Ajay Kumar Garg Engineering College, Ghaziabad. She has published over 20 papers in various national/international journals. She is a reviewer of many international journals/ conferences. Her research interest includes software evolution, machine learning, and data analytics.

Lipika Goel is a Ph.D Research Scholar from Amity University, Noida. She is currently working as an Assistant Professor in Ajay Kumar Garg Engineering College, Ghaziabad. She has 3 years of industry experience and 6.5 years of teaching experience. Her research area includes software engineering, machine learning and software reliability.

Abhay Kumar Agarwal is presently working in KNIT, Sultanpur, India as an Assistant Professor, in the Department of CSE and has total teaching experience of more than 17 years. I have completed my B.TECH degree in 1999, PGDCA in 2001, M. Tech degree in 2006 and PhD in 2017. 